\title{
Review on an Advanced High-Temperature Measurement Technology: The Optical Fiber Thermometry
}

\author{
Y. B. Yu and W. K. Chow \\ Department of Building Services Engineering, The Hong Kong Polytechnic University, Hunghom, Kowloon, Hong Kong \\ Correspondence should be addressed to W. K. Chow, beelize@polyu.edu.hk
}

Received 30 December 2008; Accepted 7 October 2009

Recommended by Shou-Shing Hsieh

Optical fiber thermometry technology for high-temperature measurement is briefly reviewed in this paper. The principles, characteristics, recent progresses and advantages of the technology are described. Examples of using the technology are introduced. Many blackbody, infrared, and fluorescence optical thermometers are developed for practical applications.

Copyright (C) 2009 Y. B. Yu and W. K. Chow. This is an open access article distributed under the Creative Commons Attribution License, which permits unrestricted use, distribution, and reproduction in any medium, provided the original work is properly cited.

\section{Introduction}

Temperature measurement is very important in fire studies and combustion research. Thermocouples are commonly used as their operation principle is simple and easy to use. However, there are some limitations [1] for thermocouple measurement in maximum measurable temperature, response, accuracy, stability, and service-life. Optical fiber thermometers (OFTs) [2-25] have been widely developed in the past decades. There, temperature is measured by photonic signals. These devices have the potential to replace the existing temperature measurement techniques or extending the measurement capabilities. This is because OFTs have the advantages of long-term stability, being unaffected by electromagnetic interference, high sensitivity, quick response, and can sustain under harsh environmental conditions.

Of all the developed OFTs, blackbody and fluoroscopic sensors are widely used. Blackbody sensors consist of a hightemperature optical fiber with an opaque cavity attached to the sensing tip. The spectral radiative flux detected at the end of the fiber is related to the temperature of the cavity via Planck's law. Temperature is obtained by measuring the spectral intensity or the intensity distribution.

Fluoroscopic sensors have a photo-luminescent material attached to the active end of an optical fiber. The sensing tip is activated by an excitation pulse from a pulsed laser or flash lamp. Temperature can be deduced from the intensity and decay-time (which depends on temperature) of the photoluminescent signal. Fluoroscopic sensors are very sensitive, but their temperature ranges are limited by the properties of the material. In general, the fluorescence intensity is weak at high temperatures due to the quenching effect. Meanwhile, the background of blackbody radiation becomes stronger at high temperature. Therefore, the signal-to-noise ratio (SNR) is poor, limiting application to high-temperature measurement.

Blackbody sensors can operate over a wide range of temperatures in principle. However, the signal intensity is weak in the lower-temperature region as the radiance intensity is nearly exponentially related to temperature. The thermometer has a strong radiance signal and higher resolution at the higher temperature region. Therefore, blackbody sensors are generally used in high-temperature applications. The optimum measuring temperature of blackbody OFT sensor varies normally from 500 to $1900^{\circ} \mathrm{C}$, although it was claimed that the temperature range could be extended down to $300^{\circ} \mathrm{C}$.

To widen the measuring range, a complex OFT scheme $[12,14]$, combining the advantages of both the blackbody and fluorescence OFT, has been developed. On the other hand, many investigations [15-21, 26] on fluorescence materials for high-temperature sensing were carried out. A study [21] on the fluorescence-based thermometry of fiber optic ruby crystal probe exposed to high temperatures was 
reported. The results showed that the single-crystal probe (made from sapphire fiber with $\mathrm{Cr}^{3+}$-doped) was able to operate continuously at temperatures up to $1400^{\circ} \mathrm{C}$. A wide temperature measurement range from $0^{\circ} \mathrm{C}$ to $1400^{\circ} \mathrm{C}$ can be covered, with uncertainties of approximately $\pm 7^{\circ} \mathrm{C}$.

In this article, blackbody, fluoroscopic and non-contact infrared OFTs will be reviewed. It is suggested that OFTs have potential advantages to be used in studying fire and combustion.

\section{Blackbody OFT}

2.1. Theoretical Background of Blackbody OFT. Blackbody OFT is based on Planck's radiation law that describes the spectral distribution of radiance for an ideal blackbody. The radiant power $\left(W_{\lambda}\right)$ emitted per unit surface area and per unit wavelength is given by

$$
W_{\lambda}=\frac{C_{1}}{\lambda^{5}} \frac{1}{\exp \left(C_{2} / \lambda T\right)-1},
$$

where $C_{1}=3.7418 \times 10^{-16} \mathrm{Wm}^{2}\left(\rightleftarrows 1.4388 \times 10^{-2} \mathrm{mK}\right)$ is the first radiation constant; $C_{2}=1.43879 \times 10^{-2} \mathrm{mK}$ is the second radiation constant; $\lambda$ is the wavelength, and $T$ is the absolute temperature in Kelvin $\mathrm{K}$.

The above equation applies to ideal blackbody radiation only. In most practical cases, the emissivity of energy radiated is corrected by a factor $\varepsilon$ (emissivity). The radiation used for measurement is observed over a narrow wave-band $\Delta \lambda$ centered at $\lambda_{0}$, and therefore the total radiated power detected $W(T)$ at a given temperature $T$ is

$$
W(T)=\frac{C_{1}}{\lambda_{0}^{5}} \frac{1}{\exp \left(C_{2} / \lambda_{0} T\right)-1} \varepsilon \Delta \lambda S \delta,
$$

where $S$ is the area of the observed radiation surface, and $\delta$ is the sight path factor.

The sight path is mainly formed by an optical fiber, which is immunised to the impact of the surrounding atmosphere observed in the open path pyrometer, that is, refractive changes in air and other disturbing effects. The fiber bending and connecting points would give transmission loss and hence errors in $\delta$.

In addition, the total emission is measured by some radiation thermometers to get the temperature based on the Stefan-Boltzman law,

$$
W_{\text {Total }}=\varepsilon \sigma T^{4},
$$

where $\sigma$ is the Stefan-Boltzman constant $\left(\sigma=5.6687 \times 10^{-8}\right.$ $\left.\mathrm{W} \cdot \mathrm{m}^{-2} \cdot \mathrm{K}^{-4}\right)$.

However, most of the photodetectors have limited response wavelength region. Correction to the above equation is required in calibrating the instrument.

By plotting $W_{\lambda}$ against wavelength for different temperature following Planck's law, it is observed that with increasing temperature, more energy is emitted and the emission peak shifts towards the shorter wavelength. The peak-emission wavelength $\lambda_{\max }$ (in $\mu \mathrm{m}$ ) is given by Wien's displacement law:

$$
\lambda_{\max }=\frac{2898}{\mathrm{~T}},
$$

where $T$ is in unit of $\mathrm{K}$.

A typical inexpensive silicon photodiode, with its long wavelength response cutting off at $\sim 1.1 \mu \mathrm{m}$, is very suitable to detect the radiation emitted from hot bodies at temperatures from 450 to $2000^{\circ} \mathrm{C}$. The use of other photodetector materials such as germanium and lead sulphides can extend the measurement to longer wavelengths. This has the potential to measure lower temperatures, but at lower sensitivity. In general, the radiation thermometry is more effective and easier to use for measuring high temperature. For lowtemperature measurement, there will be problems due to the influence of environmental temperature and sight path on the thermometers.

2.2. Configurations of Blackbody OFT. A typical blackbody OFT sensor system consists of three basic elements: an optical signal generator (the blackbody cavity), an optical-signaltransmitting system including one or more transmitting optical fibers, and an optical detecting system, as illustrated in Figure 1.

The optical signal generator is the temperature sensor which transfers thermal signals to optical ones. This is a quasi blackbody cavity at the tip of the high-temperature fiber. It is constructed with a thin ( 3 to $5 \mu \mathrm{m}$ thick) platinum (or iridium) film sputtered on a sapphire $\left(\mathrm{Al}_{2} \mathrm{O}_{2}\right)$ rod tip. The surface is coated with a protective thin film of aluminium oxide. Sapphire is transparent and nonemitting in the visible and near infrared range up to its melting point $\left(2070^{\circ} \mathrm{C}\right)$. The response of the thin film of aluminium oxide platinum to the temperature changes of the surrounding circumstance is expected to be rapid because of the very low thermal conductivity of sapphire [4]. The emissivity $\varepsilon$ of the quasi blackbody cavity is not the ideal constant of 1 , but is a function of wavelength and temperature. For example, the change in the emissivity of a cavity (with a length-todiameter ratio of 2) is less than 0.01 for $\lambda_{0}$ from 0.5 to $0.7 \mu \mathrm{m}$ and $T$ from 600 to $1300^{\circ} \mathrm{C}$. The optical-signal-transmitting system consists of the high- and low-temperature optic fibers, which transmit the signals to the detector. In the signal transmission, there are some losses due to the reflection at the ends of the fibers, misalignment of fiber coupling [27], and absorption in the fibers $[28,29]$. Therefore, a sight path factor $\delta$ is needed to correct the measurement.

The principal components of the detector system are a light gathering lens, a narrow band filter, and a photodiode or photomultiplier that converts the radiation signals to electric ones. These three elements construct a basic optical fiber thermometer. For a practical temperature measurement system, there is an electronic signal and data processing system including signal amplification, A/D conversion and PC data processing. Such an electronic system is required to be able to measure the weak signal of $7 \times 10^{-12} \mathrm{~W}$ with stable output. In general, this system has a high-impedance preamplifier combined with a high $\mathrm{Q}$ frequency-selection amplifier. 


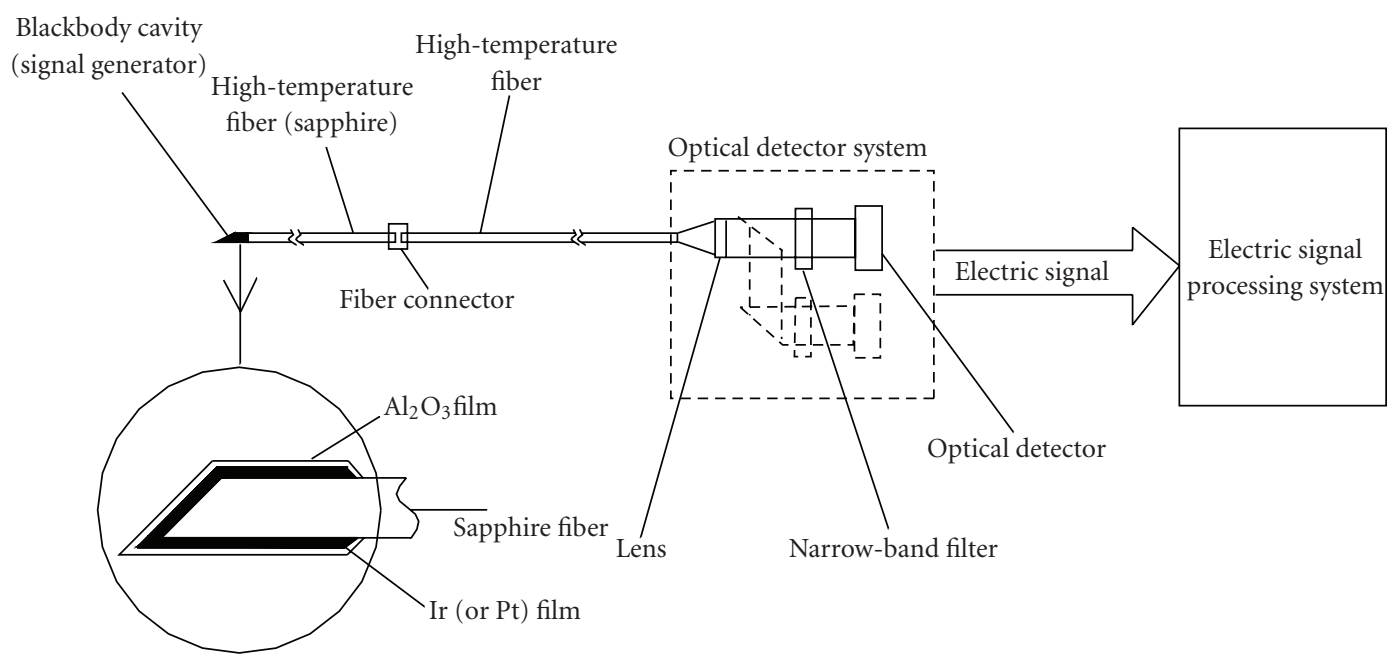

FIgURE 1: A schematic diagram for high-temperature optical fiber sensor system (from [4]).

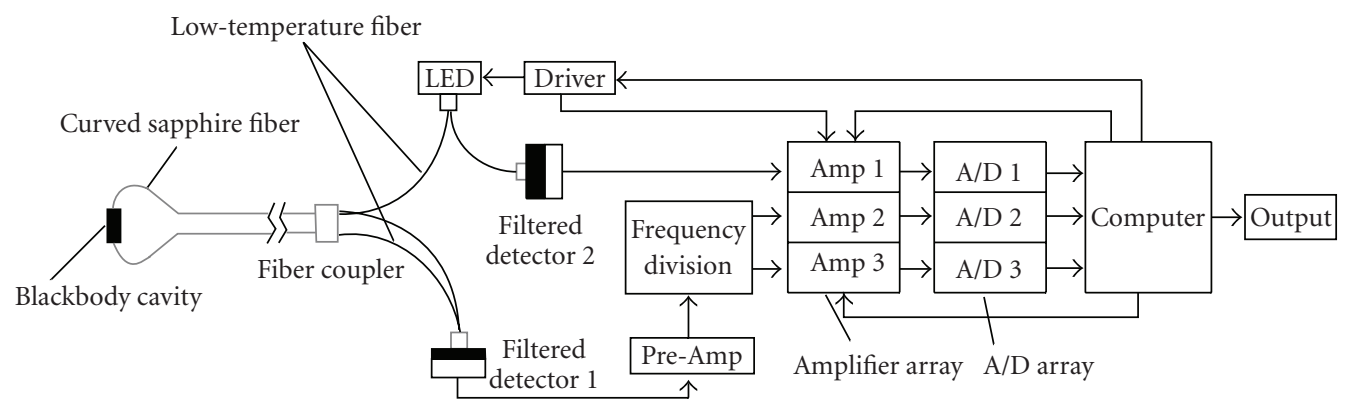

Figure 2: The configuration of a curved sapphire optic fiber thermometer (from [14]).

The optical fiber thermometer mentioned above has many advantages, such as high accuracy, intrinsic immunity to electromagnetic inference, and long lifetime, which make it very successful in various applications. Some of these excellent performances cannot be achieved by other pyrometers [30-32]. However, these sensors have their own shortcomings. One of them is that the measurement depends on some system parameters such as emissivity $\varepsilon$ and sight path factor $\delta$. Although these parameters can be determined by proper calibration, the surface of sapphire fiber is easily contaminated and corroded by impurities while working under robust environment at high-temperature. This gives unacceptable scattering loss to the fiber, reducing the transitivity of the fiber, and thus causing the instability of the sensor with single-band method, in which temperature is determined directly by the intensity of one radiation signal. In addition, couplers used to link the high-temperature sapphire fibers and the low-temperature optical fibers give unstable optic losses to the system. These are the main reasons of not using the single-band system to get higher stability. The dual-band compensation methods are better to solve this problem. Temperature is determined by the ratio of two radiation signals transmitted through the same optical system to eliminate the unstable factors. But the sensor is not so sensitive as a single-band system, due to the dividing of two nearly similar signals.
An improved method was proposed by Zhang et al. [12] to solve the problems. Temperature is determined by the ratio of the measured radiation $W(T)$ to the reference radiation $W\left(T_{r}\right)$ at an appropriate temperature $T_{r}$ recorded during a calibration process based on the following formula:

$$
\mathbf{T}=\frac{\mathrm{T}_{\mathbf{r}}}{1-\left(\lambda \mathrm{T} / \mathrm{C}_{2}\right) \ln \left[\mathrm{W}(\mathrm{T}) / \mathrm{W}\left(\mathrm{T}_{\mathbf{r}}\right)\right]},
$$
(2))

This equation is deduced from the ratio expression (see

$$
\frac{W(T)}{W\left(T_{r}\right)}=\frac{\exp \left(C_{2} / \lambda T_{r}\right)-1}{\exp \left(C_{2} / \lambda T\right)-1} \approx \exp \left[\frac{C_{2}}{\lambda}\left(\frac{1}{T_{r}}-\frac{1}{T}\right)\right],
$$

where the approximation $\exp \left(C_{2} / \lambda T\right)-1 \rightleftarrows \exp \left(C_{2} / \lambda T\right)$ is made, which is in general valid (wavelength used in most temperature measurements is 0.7 to $20 \mu \mathrm{m}$ [25]).

Another improved measurement method was proposed by Tong et al. [14] to eliminate the light path effect. A curved sapphire fiber probe was used together with a modulated light-emitting diode (LED) as shown in Figure 2. The radiation energy emitted from the blackbody cavity is transmitted through the two legs of the curved sapphire fiber and then coupled to a silica fiber splitter. The output of the fiber splitter is coupled to a narrow-band filtered photodetector 1 . The reference signal that comes from a $2.0 \mathrm{kHz}$ 


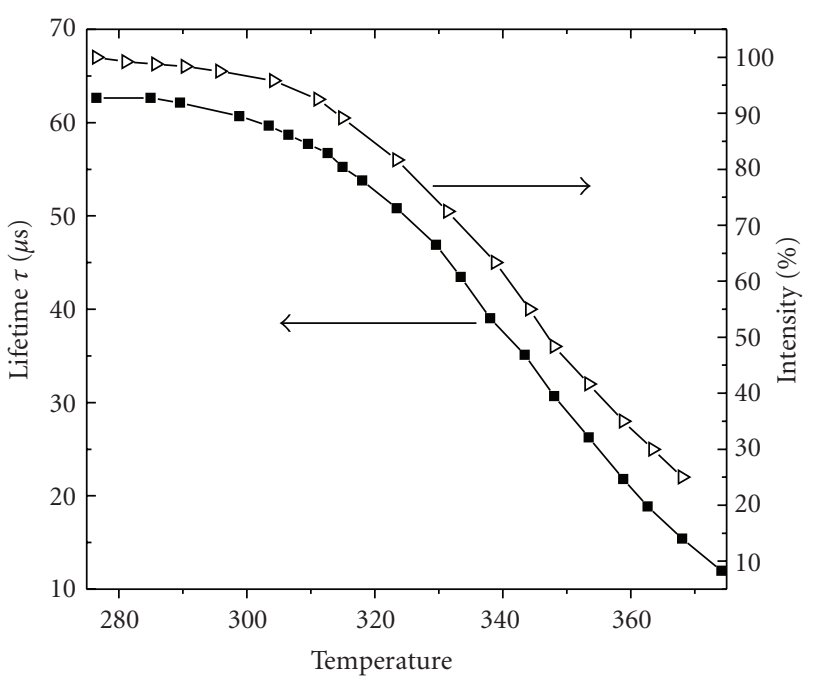

Figure 3: Thermal characteristics of $\mathrm{Cr}^{3+}$ fluorescence in LiSAF. The solid squares and open triangles represent the data on fluorescence lifetime and intensity, respectively (from [15]).

modulated infrared LED is separated into two beams by a fiber splitter, one to the sapphire fiber and the other to a monitoring photo-detector 2 . After transmitting through the whole length of the sapphire fiber, the reference signal goes into the silica fiber and then to the filtered detector 1 . There, the two signals are converted to a combined electrical signal which is amplified by a preamplifier. The output of the preamplifier is then sent to a frequency division circuit where the combined signal is separated into two electrical signals that correspond to the radiation and the reference signal. The two signals are processed individually by the amplifier array and A/D array. The ratio of intensities of the two signals can then be worked out by the computer to give the value of temperature.

2.3. Application of Blackbody OFTs. Blackbody OFTs have been proved to be very successful in measuring high temperatures [22-25] in basic metals, glass productions, and the initial hot forming processes for such materials. Boiler burner flames, tube temperatures, and critical turbine areas are typical applications in power generation operations. Rolling lines in steel and other fabricated metal plants also pose harsh conditions, which are well handled by fiber optics. Typical applications include temperature measurements in all types of furnaces, combustion inside engines, sintering operations, ovens, and kilns. Automated welding, brazing, and annealing equipment often generate large electrical fields, which can disturb conventional sensors.

High-temperature processing operations in cement, refractory, and chemical industries often use fiber optic temperature sensing. At somewhat lower temperatures, plastics processing, papermaking and food processing operations are making more use of the technology. Fiber optic temperature sensors are also used in fusion, sputtering, and crystal growth processes in the semiconductor industry.

\section{Non-Contact Infrared Thermometers}

Another type of OFTs is non-contact infrared (IR) thermometer. Temperature measurements can be classified into two types: invasive and noninvasive, or contact and noncontact. The first type requires direct contact between the measurement device and the specimen, as thermocouple temperature measurement system. The blackbody OFT mentioned above is one kind of contact thermometers. No direct contact is required for the second type. The measured specimen can be observed at distance away from the instrument.

The contact instrumentation must be able to stand the high temperature concerned. In high-temperature or chemically reactive applications such as flames or plasmas, invasive instrumentation can degrade with time. If operating above the material limits, it would be disintegrated completely. On the other hand, thermal probes are needed in most contact thermometers. A thermal equilibrium state between the probes and the measured specimen should be reached while measuring the temperature, and therefore this confines the response of the OFTs. Noninvasive methods are not bound by these constraints. In addition, noninvasive instrumentation can be useful in determining the temperature of moving components without any additional telemetry systems. Both temperature measurements at a point and the variation over a region, by scanning, can be made. Most noninvasive techniques measure temperature from the electromagnetic spectrum emitted by the measured target, so are in fact radiometers. Infrared devices are sensitive to that part of the spectrum, and infrared thermometers are the most popular non-contact temperature measurement instruments.

Infrared thermometry is based on the same theoretical background as the blackbody OFT, that is, the blackbody emission law. But the blackbody cavity is not used in the infrared thermometry. The detected emission comes directly from the surfaces of those measured targets. There is not a blackbody cavity at the end of the IR OFT, and the end is placed away from the measured target. So, no hightemperature fibers are needed. However, in comparing with the blackbody OFT, the effects of emission and path factors and environment on the measurements are greater, and the accuracy is lower. With the rapid development of the electronic data processing technology and the measurement methods, the performance of the infrared thermometers is greatly improved.

\section{Fluorescence-Based OFT}

Fluorescence-based OFT is based on the temperaturedependent fluorescence decay time or fluorescence intensity of the appropriate materials. Figure 3 shows the temperature-dependence of fluorescence lifetime and intensity data measured from $\mathrm{Cr}^{3+}$ doped in $\mathrm{LiSrAlF}_{6}$ (LiSAF).

Most of the earliest fluorescence OFT systems are based on the fluorescence intensity of materials. An early commercial system utilized europium-activated lanthanum and gadolinium oxysulphide as alternate sensor materials [33]. The fluorescence from these rare-earth phosphors consists 
of sharp line originating from different excited states of the trivalent europium ion. Since the relative populations of theses excited states after excitation are strong function of temperature, the relative intensities of the emission lines are also quite temperature-dependent. By measuring the intensities of the two lines originating from different excited states, the system derives the temperature from the ratio of the line intensities observed. In another early fluorescence thermometer system [34], the sensor is a small crystal of gallium arsenide sandwiched between gallium aluminium arsenide layers. The sensor is caused to luminesce by radiation from a gallium arsenide light-emitting diode (LED). As the temperature of the sensor is raised, the luminescence broadens and shifts to longer wavelength. Portions of the luminescence are transmitted to the optic fibers with adjacent pass band, the intensity in each band is measured, and the temperature can be obtained from the ratio of the two intensities.

Although a number of intensity-based fluorescence thermometry systems are available, it can be seen that the technique has certain limitations in performance and cost [10]. On the other hand, the thermometric technique based on the measurement of fluorescence lifetime was developed $[5,7,8]$, and has been more widely used in the development of commercial fluorescence thermometers to date. In this article, only the lifetime-based fluorescence thermometers are described.

Two main methods are used in the measurement of fluorescence decay time, namely the "pulse method" and the "phase detection method." In the first method, the sample is excited by short pulse of light and the resulting emission in the longer wavelength is an exponentially decaying function whose rate of decay can be measured. In the second method, the sample is excited with sinusoidally modulated light, which results in a sinusoidal fluorescent emission that lags in phase with the original excitation sinusoidal light. This phase shift gives an indication of the decay time. The major advantage of such decay-time measurement techniques is that they obviate the need for accurate measurements of the input light intensity for reference purposes, a very important consideration in fiber-optical sensors.

4.1. Pulse Measurement of Fluorescence Lifetime. The common feature of the schemes in this method is that the excitation light applied to the fluorescence material is a high intensity $\delta$-function pulse (e.g., a laser pulse or that from a flash lamp) or a rectangular pulse, and the measurement is derived from the observation of the fluorescence decay after the removal of the excitation light. Some typical schemes of this type which have been used in thermometry applications are outlined in the following.

4.1.1. Two-Point Time Constant Measurement. This is a very straightforward method which was used by several groups in the early stage of the development of fluorescence OFT systems $[7,35,36]$. The fundamental principle of this technique is illustrated in Figure 4. The approach is to compare the intensity level at two points along the exponential decay

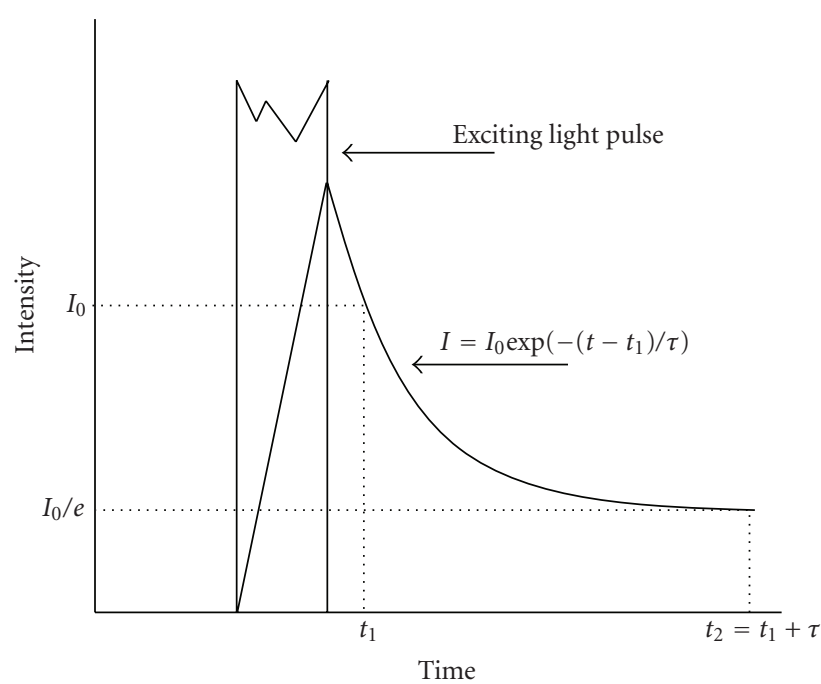

FIGURE 4: The principle of two-point time constant measurement technique.

curve after the excitation pulse has terminated. The circuitry employed to do this is designed to measure the first value of the decaying signal $I_{0}$ that occurs at a fixed time $t_{1}$ after the termination of the pulse. A second intensity level of value $I_{0} / e$ is then calculated and established as a reference. When the decaying signal falls to that level, the time $t_{2}$ at which "crossover" occurs is noted. The difference $t_{2}-t_{1}$ is the time constant $\tau$ of the exponentially decaying signal. In most cases, the fluorescence decay process observes a single or "quasi-single" exponential law, such as that shown in Figure 4. Thus, the time constant $\tau$ may be used as the fluorescence lifetime. Then, the temperature of the sensor can be obtained directly with reference to an empirical "lookup" table stored within the instrument.

This type of method is simple and inexpensive in relation to the electronic components used. Since the fluorescence signal is measured after the excitation pulse is over, the detector optics do not have to be designed to discriminate strongly against stray signal from the excitation source. However, a significant disadvantage of this method is that the signal is only measured at two special times, and as a result, the precision is greatly limited.

The two-point measurement technique was used in the system reported by Wickersheim and Sun [36], where a lamp phosphor, tetravalent manganese activated magnesium fluorogermanate, was incorporated as the fluorescent sensor itself. With a xenon flash lamp used as the excitation source, the observed lifetime ranged from $0.5 \mathrm{~ms}\left(\right.$ at $450^{\circ} \mathrm{C}$ ) to $\sim 5 \mathrm{~ms}$ $\left(-196^{\circ} \mathrm{C}\right)$. It was reported to have an accuracy of $2^{\circ} \mathrm{C}$ over the whole range without calibration of the instrument, and $0.2^{\circ} \mathrm{C}$ accuracy with a single point calibration.

4.1.2. Integration Method. To achieve higher precision from the pulse measurement approach, several techniques have been developed based on the integration of the decaying fluorescence signal over different periods of time. One example of this method is the signal-processing scheme 
used by Sholes and Small [5] in their early nonfiber study of ruby fluorescence decay. As in the two-point method, the measurement process is started when the decaying fluorescence falls below a preset level (set as $t=0$ ). The signal is integrated over the two fixed periods, $T 1$ and $T 2$, as illustrated in the following:

$$
\begin{aligned}
& \mathrm{A}=\int_{0}^{\mathrm{T}_{1}}(\text { decay }+ \text { noise }) ; \quad \mathrm{B}=\int_{0}^{\mathrm{T}_{2}}(\text { decay }+ \text { noise }) \\
& \mathrm{C}=\int_{\mathrm{T}_{0}}^{\mathrm{T}_{0}+\mathrm{T}_{1}}(\text { noise }) ; \quad \mathrm{D}=\int_{\mathrm{T}_{0}}^{\mathrm{T}_{0}+\mathrm{T}_{2}}(\text { noise }),
\end{aligned}
$$

where $\mathbf{T}_{\mathbf{0}}$ is the preset time at which the signal has decayed to zero. Therefore, the fluorescence lifetime $\boldsymbol{\tau}$ is obtained by solving the implicit relation:

$$
\frac{\mathrm{A}-\mathrm{C}}{\mathrm{B}-\mathrm{D}}=\frac{1-\exp \left(-\mathrm{T}_{1} / \tau\right)}{1-\exp \left(-\mathrm{T}_{2} / \tau\right)}
$$

Another example is the balanced integration method described by Sun [37]. There, a dual-slope integrator is used to balance the integrals of two sequential areas under the decay curve,

$$
A_{1}=\int_{t_{1}}^{t_{2}}(\text { decay }) ; \quad A_{2}=\int_{t_{2}}^{t_{3}}(\text { decay }) .
$$

The first integration is carried out over a fixed time interval between the predetermined time $t_{1}$ and $t_{2}$ after the pulse has terminated. Upon completion of the first integration, the polarity is reversed and negative integration (de-integration) begins. This continues until the second integrated area exactly equals the first, and the combined integral is zero. This technique was designed to achieve $0.01^{\circ} \mathrm{C}$ resolution using the same sensor material that had been used in the two-point measurement by Wickersheim and Sun as mentioned above.

4.1.3. Digital Curve Fit Method. Another modular system, WTS-11, introduced by Luxtron company, was designed to monitor winding temperature in power transformer [38], utilizing a technique which takes advantage of the highspeed digital signal processor (DSP). This technique has been described in detail by Sun [37], and is called "digital curve fit method" $[26,39,40]$.

In this system, the excitation spectrum of the fluorescence material allows the use of a convenient excitation source, for example, red LED or laser diode. When the sensor is excited by sequential light pulses from an LED or laser diode, a periodic decaying luminescent signal is resulted. A selected portion of each decay curve is digitalized, after the detected signal has passed through a low noise and wide bandwidth amplifier. The digital samples, after correction for any offset, are then processed by the DSP to provide the best exponential decay curve by means of the least squares curve fitting technique. The exponential is first converted to a straight line by taking the natural logarithm of the digitised signal. The slope of the best fit straight line is proportional to the lifetime of the luminescence. The results of a number of curve fits are averaged further to reduce the effect of noise. The average lifetime is then compared with the values stored in a digital look-up table to determine the temperature of the sensor.

4.2. Phase Detection Schemes for Fluorescence Lifetime Measurement. As mentioned earlier, the fluorescence sample is excited with sinusoidally modulated light, which results in a sinusoidal fluorescent emission that lags in phase with the original excitation sinusoidal light. It can be shown that the phase difference between the input light and the excited fluorescence optical signals is

$$
\varphi=\arctan (2 \pi f \tau)
$$

where $f$ is the frequency of the fluorescence optical signals, and $\tau$ is the time constant of the fluorescence decay, or lifetime. Thus, the fluorescence lifetime can be determined from a measurement of the phase shift. This method is of high accuracy and is meant to be used in precision measurement instrumentation, since it is inherently insensitive to dc-offset and ac-noise in the sinusoidal signal. The latter can be substantially reduced by a great variety of electronic devices, ranging from various electronic analog filters and digital filters to the lock-in amplifiers.

4.2.1. Phase-Locked Detection of Fluorescence Lifetime. During the early stage of development, the lack of convenient and economic excitation modulation schemes limited the use of the phase shift technique in fluorescence thermometry. Now, with the wide availability of cheap and easily modulated high-power LEDs or laser diode, this technique has found its wide application in the thermometry area.

A signal-processing scheme, phase-locked detection of fluorescence lifetime [41] (PLD), has been developed for the achievement of a simple, inexpensive, and versatile electronic scheme for the detection of fluorescence lifetime, and successfully applied to several fiber optic thermometer schemes [11-13, 21, 41]. A schematic of the phase-locked detection system is shown in Figure 5. The excitation light source is intensity-modulated sinusoidal or square-wave signal generated from a voltage-controlled oscillator (VCO). If the excitation light source is sinusoidal or square-wave signal, the system is called PLD with analog modulation of excitation source and single reference signal (PLD-AMSR) as shown in Figure 5(a), or PLD with pulse modulation and a single-reference signal (PLD-PMSR) as shown in Figure 5(b). The fluorescence signal from the sensor, which follows the variation of excitation light in intensity but lags by a phase shift $\varphi$, is sent to a lock-in amplifier to mix with a reference signal $\left(v_{r}\right)$ derived from the VCO output with a phase lag of a fixed fraction $\alpha$ of the period. The resulting mixed signal is then filtered by a low-pass filter (LPF) and further integrated, with the resultant voltage signal being fed back to control the output frequency of the VCO. The VCO will finally be driven to operate at a frequency at which the integration of the mixed signal is zero. The period $(\tilde{T}=2 \pi / \omega)$ corresponding to the frequency is directly 


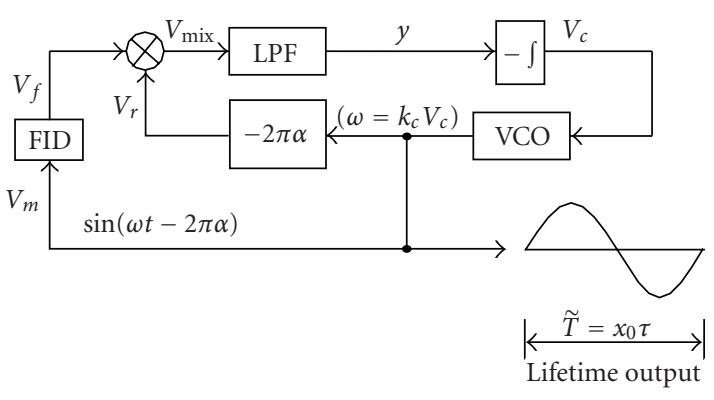

(a)

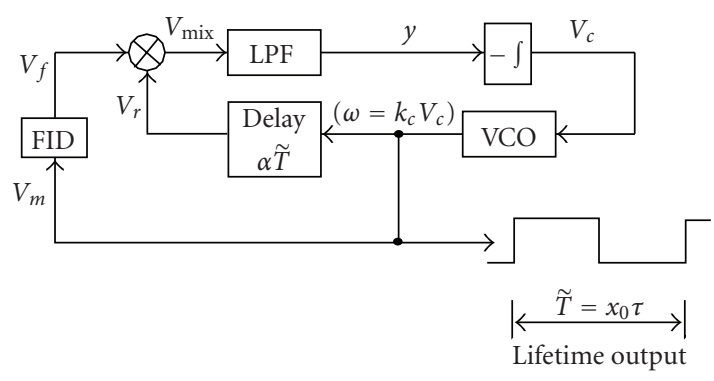

(b)

Figure 5: Phase-lock detection system for fluorescence lifetime using single reference signal. (FID: the fluorescence inducing and detecting devices, $v_{m}$ : signal to modulate the output intensity of the excitation light source, $v_{f}$ : the fluorescence signal, $v_{r}$ : the reference signal, LPF, low-pass filter, VOC: voltage-controlled oscillator) (from [41]).

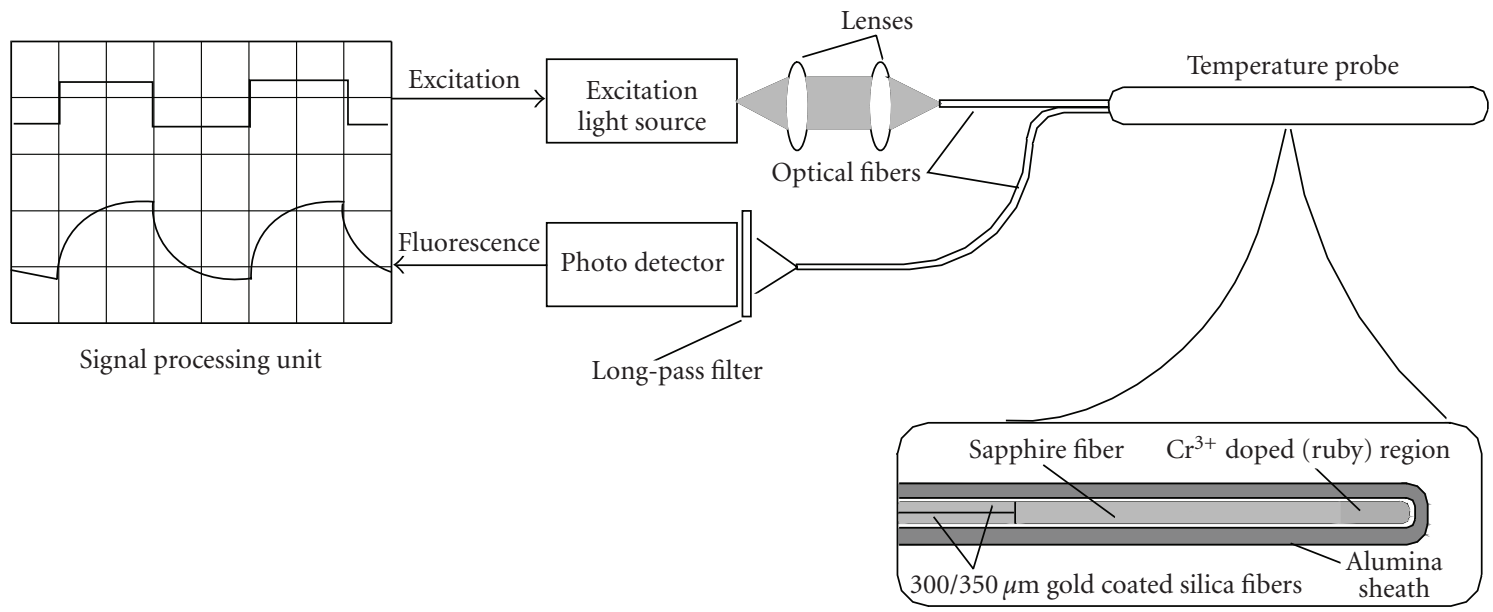

Figure 6: A schematic diagram of the fluorescence lifetime measurement system, with an enlarged view of the fluorescent fibre optic probe (from [21]).

proportional to the lifetime. This allows a high resolution to be achieved over a wide range of lifetime. In such systems, while the frequency varies with the fluorescence lifetime, the phase shift between the excitation and fluorescence signals is always kept at a constant, and determines the reference decay ratio $\alpha$.

\subsubsection{Two Examples of Lifetime-Based Fluorescence Thermometers}

(i) Sapphire-Ruby Single-Crystal Fiber Thermometers. A fluorescence lifetime measurement system recently developed by Grattan et al. [21] for use in OFT is shown Figure 6. In this system, a laser diode (LD) with $5 \mathrm{~mW}$ optical output at a wavelength of $635 \mathrm{~nm}$ is used as the light source, well suited to the excitation of the ruby fluorescence, and coupling conveniently to the absorption bands in the material. About $60 \%$ of the LD output light is actually launched into the sapphire-ruby fiber through a $300 / 350 \mu \mathrm{m}$ gold-coated silica fiber, well suited to withstand high temperatures. The ruby fluorescence induced is collected by another gold-coated silica fiber, as is shown in the figure, and delivered to the photodetector where a long-pass filter with a $50 \%$ cut-off wavelength at $690 \mathrm{~nm}$ is used to block the reflected excitation light at $635 \mathrm{~nm}$. The photodetector employed is a silicon PIN photodiode with a $1 \mathrm{~mm}^{2}$ sensitive area, and thus the fluorescence emitted can be monitored well over the $690 \mathrm{~nm}$ spectral region. The output of the excitation light source is modulated in an "on-and-off" manner. At the same time, the induced fluorescence decay signal is processed in a signalprocessing unit based on the PLD-PMSR technique, which then produces a measurement of the fluorescence lifetime.

The temperature probe of this system is a singlecrystalline sapphire fiber with $\mathrm{Cr}^{3+}$-doped (ruby) tip, as shown in Figure 6. Such fibers have been found to be of high optical quality, mechanically stable and able to withstand very high temperatures, particularly in comparison to silica fibers, as their melting point is in the region of $2000^{\circ} \mathrm{C}$. Thus, it is important for stable operation of this type of fiber that a series of tests of the integrity of the essential fiber were carried out. The fiber sample had been subjected to long-term heat treatment tests at a range of very high temperatures, including $1400^{\circ} \mathrm{C}$ and $1500^{\circ} \mathrm{C}$, respectively. The results of such tests indicate the reproducibility of the thermal characteristics of the fluorescence from the sensing element, where the $\mathrm{Cr}^{3+}$-doped 


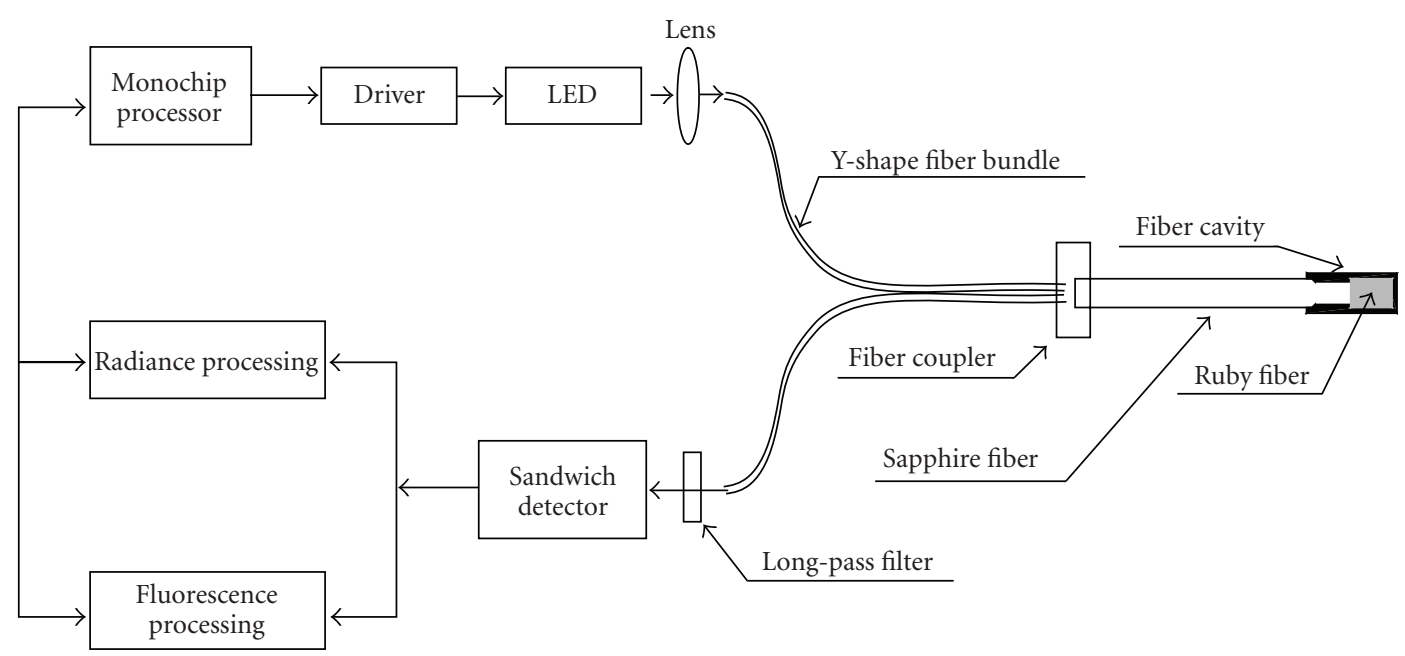

Figure 7: A systematic diagram of a cross-referencing OFT (from [42]).

region of the single-crystal sapphire fiber shows that the sapphire-ruby single-crystal probe can operate continuously at temperatures up to $1400^{\circ} \mathrm{C}$. With the technique of crossreferencing between fluorescence and blackbody radiation $[12,42]$, the probe is able to cover a wide temperature measurement range extending from sub-zero temperatures to $1400^{\circ} \mathrm{C}$.

(ii) Cross-Referencing OFT. As mentioned before, the fluorescence intensity is weak at high temperatures owing to the quenching effect, and meanwhile the background of blackbody radiation becomes stronger. Then the SNR is poor, which limits the high-temperature application of this scheme. The blackbody sensors are generally used in high-temperature applications. To enlarge the measuring range, a complex OFT scheme [12, 14], which combines the advantages of both the blackbody and fluorescence OFT, has been developed based on the cross-referencing between the fluorescence-lifetime and blackbody-radiation measurements, and is called the cross-referencing scheme.

Here, an example of the cross-referencing OFT, which was developed by Shen et al. [42], is presented. The system construction is quite similar to that using only the fluorescent decay, but a signal-processing scheme of thermal radiance was added. A periodically modulated green super-bright LED was used as the exciting light source. The exciting light was coupled into the small branch of the Y-shaped fiber bundle by a condenser lens as shown in Figure 7. The big branch of the Y-shaped fiber bundle was directly coupled with the sapphire fiber. Another small branch of the Y-shaped fiber bundle transmitted the fluorescence or the radiance signal to the detector. For convenience and efficiency, a sandwich two-wavelength detector was used in the system, which combined the Si and InAsGa detectors together into one package. The spectral response centers of the detector were 900 and $1600 \mathrm{~nm}$. The Si detector centered at $900 \mathrm{~nm}$ is essential for the fluorescence detection of the ruby centered at $694.3 \mathrm{~nm}$. For filtering out the exciting LED light from the signal, a long-pass filter from $670 \mathrm{~nm}$ was used before the detector. After the detector and the preamplifier, there was a two-channel selection switch, which corresponded to the signal processing schemes of using the radiance intensity and the fluorescent lifetime detection, respectively. This selection was controlled by a processor, depending on the ambient temperature and the thermal radiance detected. For example, if the ambient temperature is over $400^{\circ} \mathrm{C}$ and the radiance signal is strong enough, the radiance intensity detection scheme is used while the LED is turned off. Otherwise, the LED continues working, and the fluorescent lifetime detection scheme is used. The thermal probe of the system is a sapphire fiber grown from the laser-heated pedestal growth method. Its end part is doped with $\mathrm{Cr}^{3+}$ ion and coated with some radiance material to constitute a mini-fiber cavity. This system was claimed to have a large measurement range from 20 to $1800^{\circ} \mathrm{C}$.

\section{Advantages of OFTs over the Traditional Thermometers}

The fundamental differences between an optical fiber and a metal wire for signal transmission give OFTs the following advantages [10] over traditional thermometers.

5.1. Electrical, Magnetic, and Electromagnetic Immunity. The materials in the OFT probes are typically good electrical insulators. Since they do not conduct electricity, the probes cannot (in principle) introduce electrical shorting path or cause electrical safety problems. Likewise, they do not absorb significant amount of electromagnetic radiation or become heated by such fields. In addition, stray fields cannot induce electrical noise in fibers, so the probes exhibit a very high level of electromagnetic immunity.

5.2. Small Sensor Size and Rapid Response. Since the typical sensor is as small as in diameter with the fiber itself, the sensor, in principle, can be extremely small. This allows its use in applications such as in medicine or in microelectronics 
where size is critical, and allows a more accurate temperature distribution. Furthermore, since small size means small thermal mass, the fiber optic sensors typically exhibit a very rapid thermal response. Blackbody OFTs of $\mathrm{kHz}$ frequency response have been achieved [43].

5.3. Safety. Safety may be the main reason for the use of OFTs in some particular areas of application. Most OFTs require no electric power at the sensor end of the system. They generate their own optical signal or they are "powered" remotely by radiation from a light source located within the OFT instrument, therefore introducing no danger of electrical sparks in hazardous environments. There is a reason to believe that at normal levels of optical power coupled into fiber optics, that is, levels of up to several hundred million-watts of optical power, there is almost no hazard with any accidental fracture of cable and the possible focusing of the optical radiation by the lensing effects of the broken end. Particularly in the chemical industry, where highly explosive gases or gas mixtures may be used, this is an important consideration, but on the whole in normal use, optical sensing systems can be considered intrinsically safe.

5.4. Capability of Remote Measurement. The small size of fiber and its electrical, chemical and thermal inertness allow for long-term location of the sensor deep inside complex equipment and thereby provide access to locations which are difficulty to address, where monitoring the temperature may be of interest. Beyond this, some OFTs allow non-contact or remote sensing of the temperature, and this is easily accomplished with infrared thermometers.

5.5. Other Advantages. Besides these generic advantages, some of the optical techniques exhibit an unusually wide range of operation with precision good enough to meet reasonable requirements. At the same time, these techniques provide simplicity of calibration, or as in the fluorescence lifetime-based OFT, the absence of the need to calibrate individual probes.

\section{Comparison among Different OFTs}

As mentioned above, optical fiber thermometers have many advantages over conventional thermometers. However, in selecting a measurement method and the associated instrument among the various types of OFTs to suit a particular application, it is still necessary to make some considerations such as measurement range, sensitivity, accuracy, response, service-life, stability, contact method, and cost. A comparison among the different OFTs is presented in the following.

6.1. Measurement Range, Sensitivity, and Accuracy. Among the different OFTs, the blackbody OFT is most suitable for high-temperature measurement (up to $2000^{\circ} \mathrm{C}$ ), it has high sensitivity at high temperature because the cavity can generate an intensive optical signal. But it will lose its sensitivity at lower temperatures, so it is not suitable for lowtemperature measurement. In contrast to blackbody OFT, fluorescence thermometers are suitable for low-temperature measurement. However, the fluorescence intensity is weak at high temperatures, and the lifetime decreases with increasing temperature. At high temperature, the background of blackbody radiation becomes stronger. Therefore, the signalto-noise ratio (SNR) and sensitivity are poor. The complex OFT based on the combination of the two thermometric techniques can work in a wide temperature range, but this makes the system more complicated and expensive. Both types of the OFTs have high accuracy in their effective measurement ranges, generally less than $0.5^{\circ} \mathrm{C}$.

For non-contact IR thermometers, they are based on the same measurement principle as the blackbody OFTs, and so are better used in high-temperature measurement. Although the blackbody-radiation-based thermometer can measure lower temperatures up to room temperature, the accuracy and sensitivity are generally low. To raise the accuracy and sensitivity for low-temperature measurement, some special photodetectors, complicated signal, and data processing systems are required. This would make the systems expensive and slow in response. IR thermometers are suitable for qualitative measurement due to its low accuracy. In addition, the accuracy of IR thermometers has to be ensured by detailed knowledge of the detected targets. This makes the calibration difficult in measuring the temperatures of different detected targets.

6.2. Response. Since there are no thermal probes in noncontact IR thermometers, thermal-equilibrium processing between the probes and the measured specimen is not needed. The response speed is not confined by the properties of the thermal probe. Therefore, non-contact IR thermometers have faster response and are suitable for quick and qualitative measurements. However, the contact OFTs also exhibit quite rapid thermal response due to the small size (in the order of micrometer in diameter) of the fiber optic probes. Except for the size of the probe (or the blackbody cavity), the heat conduction between the external and inner sapphire parts has also a considerable effect on the response time of OFTs. Therefore, in the construction of the probe, the size and heat conduction of the probe should be considered seriously.

6.3. Stability and Service-Life. For blackbody-radiationbased OFT, the impact of the variance in the ambient temperature, emissivity and sight path factors would induce some instability in measurement, and a real-time calibration is required. For high temperature, this unstable impact is very small, but for lower temperature, it becomes considerable. Unlike blackbody radiation thermometer, the measurement for lifetime-based fluorescence OFT is free of any impact of the variance in ambient temperature, emissivity and sight path factors. Thus, generally, the lifetime-based fluorescence OFT provides a very stable measurement.

Both blackbody and fluorescence OFTs have a long service-life because their probes are coated with some protective films. Generally, the radiation and thermal properties of the blackbody cavity can be kept unchanged for a long time 
at high temperature. However, the fluorescent properties of OFT sensor would have some changes when it stays in high temperature for a long time, although this can be improved through some heat treatments. The non-contact IR OFT should have a longer service-life than the above two, because there is no thermal probe that contacts with the measured objects. But the measurement by non-contact IR OFT is of lower accuracy and unstable due to its strong impact of ambient temperature, emissivity and sight path factors.

In summary, in comparison with traditional thermometers, OFTs have the advantages of electromagnetic immunity, high sensitivity, small probe size and quick response, long-term stability, safety, and they can sustain under harsh environmental conditions. Among the different OFTs described above, blackbody OFTs are most suitable for high-temperature applications, fluorescence OFTs for lower-temperature measurements, and non-contact IR thermometers for the application in quick and qualitative measurements.

\section{Nomenclature}

\begin{tabular}{|c|c|}
\hline$\alpha:$ & Phase lag of the period \\
\hline$\lambda:$ & Wavelength $(\mathrm{m})$ \\
\hline$\Delta \lambda:$ & Narrow wave-band $(\mathrm{m})$ \\
\hline$\lambda_{\max }:$ & $\begin{array}{l}\text { Peak-emission wavelength given by Wien's } \\
\text { displacement law }(\mu \mathrm{m})\end{array}$ \\
\hline$\delta:$ & Sight path factor \\
\hline$\varepsilon:$ & Emissivity \\
\hline$\sigma:$ & $\begin{array}{l}\text { Stefan-Boltzman constant }=5.6687 \times 10^{-8} \\
\left(\mathrm{~W} \cdot \mathrm{m}^{-2} \cdot \mathrm{K}^{-4}\right)\end{array}$ \\
\hline$\tau:$ & Fluorescence life-time (s) \\
\hline$\varphi:$ & $\begin{array}{l}\text { Phase difference between the input light and } \\
\text { the excited fluorescence }\end{array}$ \\
\hline$\omega:$ & $\begin{array}{l}\text { Frequency of reference signal from VCO in } \\
\text { Figure } 5\end{array}$ \\
\hline$A, B, C, D:$ & $\begin{array}{l}\text { Integration of fluorescence signal over fixed } \\
\text { periods in }(7)\end{array}$ \\
\hline$A_{1}, A_{2}:$ & $\begin{array}{l}\text { Integration of fluorescence signal over fixed } \\
\text { periods in (9) }\end{array}$ \\
\hline$C_{1}$ : & $\begin{array}{l}\text { First radiation constant }=1.4388 \times 10^{-2} \\
(\mathrm{~W} \cdot \mathrm{m})\end{array}$ \\
\hline$C_{2}$ : & $\begin{array}{l}\text { Second radiation constant }=1.43879 \times 10^{-2} \\
(\mathrm{~m} \cdot \mathrm{K})\end{array}$ \\
\hline$e:$ & Base of the natural logarithms $=2.7321$ \\
\hline$f:$ & Frequency of fluorescence \\
\hline I: & Emission intensity of fluorescence \\
\hline$I_{0}:$ & $\begin{array}{l}\text { First value of decaying signal defined in } \\
\text { Figure } 4\end{array}$ \\
\hline S: & Area of the observed surface $\left(\mathrm{m}^{2}\right)$ \\
\hline$t:$ & Time (s) \\
\hline$t_{1}, t_{2}, t_{3}:$ & Selected time in Figure 4 and (9) \\
\hline$T_{1}, T_{2}:$ & Fixed periods \\
\hline$T_{0}:$ & $\begin{array}{l}\text { A preset time at which the fluorescence } \\
\text { decays to zero }\end{array}$ \\
\hline 1. & Temperature $(\mathrm{K})$ \\
\hline$T_{r}$ & Reference temperature \\
\hline & Period \\
\hline
\end{tabular}

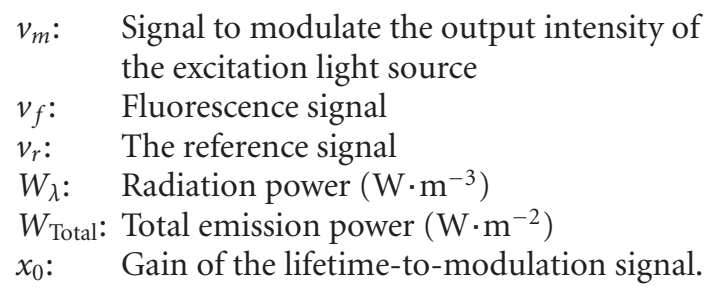

\section{Acknowledgment}

The work is supported by The Hong Kong Polytechnic University under the postdoctoral fellowship scheme (GYW68).

\section{References}

[1] W. N. Lutz, G. T. Gillies, and S. W. Allison, "Thermographic phosphors: an alternative to bare wire type $\mathrm{K}$ thermocouples at high temperatures," Industrial Heating, vol. 54, no. 10, pp. 36-41, 1987.

[2] V. C. Fernicola and L. Crovini, "Digital signal processing for fiber-optic thermometers," IEEE Transactions on Instrumentation and Measurement, vol. 44, no. 2, pp. 447-450, 1995.

[3] V. C. Fernicola and L. Crovini, "Digital optical fiber point sensor for high-temperature measurement," Journal of Lightwave Technology, vol. 13, no. 7, pp. 1331-1334, 1995.

[4] R. R. Dils, "High-temperature optical fiber thermometer," Journal of Applied Physics, vol. 54, no. 3, pp. 1198-1201, 1983.

[5] R. R. Sholes and J. G. Small, "Fluorescent decay thermometer with biological applications," Review of Scientific Instruments, vol. 51, no. 7, pp. 882-884, 1980.

[6] A. S. Gerges and D. A. Jackson, "A fibre-optic based high temperature probe illuminated by a multimode laser diode," Optics Communications, vol. 80, no. 3-4, pp. 210-214, 1991.

[7] K. T. V. Grattan and A. W. Palmer, "A fiber optic temperature sensor using fluorescence decay," vol. 492 of Proceedings of SPIE, pp. 535-542, 1984.

[8] K. T. V. Grattan, "The use of fiber optic techniques for temperature measurement," Measurement and Control, vol. 20, pp. 32-39, 1987.

[9] K. T. V. Grattan, R. K. Selli, and A. W. Palmer, "Ruby decaytime fluorescence thermometer in a fiber-optic configuration," Review of Scientific Instruments, vol. 59, no. 8, pp. 1328-1335, 1988.

[10] K. T. V. Grattan and Z. Y. Zhang, Fiber Optic Fluorescence Thermometry, Chapman \& Hall, London, UK, 1995.

[11] Z. Y. Zhang, K. T. V. Grattan, and A. W. Palmer, "A novel signal processing scheme for a fluorescence based fiber-optic temperature sensor," Review of Scientific Instruments, vol. 62, no. 7, pp. 1735-1742, 1991.

[12] Z. Y. Zhang, K. T. V. Grattan, and A. W. Palmer, "Fiber optic temperature sensor based on the cross referencing between blackbody radiation and fluorescence lifetime," Review of Scientific Instruments, vol. 63, no. 5, pp. 3177-3181, 1992.

[13] Z. Y. Zhang, K. T. V. Grattan, and A. W. Palmer, "Fiber-optic high-temperature sensor based on the fluorescence lifetime of alexandrite," Review of Scientific Instruments, vol. 63, no. 8, pp. 3869-3873, 1992.

[14] L. M. Tong, Y. H. Shen, and L. H. Ye, "Performance improvement of radiation-based high-temperature fiber-optic sensor 
by means of curved sapphire fiber," Sensors and Actuators A, vol. 75, no. 1, pp. 35-40, 1999.

[15] Z. Y. Zhang, K. T. V. Grattan, and A. W. Palmer, "Temperature dependences of fluorescence lifetimes in $\mathrm{Cr}^{3+}$-doped insulating crystals," Physical Review B, vol. 48, no. 11, pp. 7772-7778, 1993.

[16] Z. Y. Zhang, K. T. V. Grattan, A. W. Palmer, B. T. Meggitt, and T. Sun, "Fluorescence decay-time characteristics of erbiumdoped optical fiber at elevated temperatures," Review of Scientific Instruments, vol. 68, no. 7, pp. 2764-2766, 1997.

[17] Z. Y. Zhang, K. T. V. Grattan, A. W. Palmer, and B. T. Meggitt, "Potential for temperature sensor applications of highly neodymium-doped crystals and fiber at up to approximately $1000^{\circ}$ C, Review of Scientific Instruments, vol. 68, no. 7, pp. 2759-2763, 1997.

[18] Z. Y. Zhang, K. T. V. Grattan, A. W. Palmer, B. T. Meggitt, and T. Sun, "Characterization of erbium-doped intrinsic optical fiber sensor probes at high temperatures," Review of Scientific Instruments, vol. 69, no. 8, pp. 2924-2929, 1998.

[19] M. Stanciu and K. T. V. Grattan, "LiCAF crystal-based optical fiber thermometry," Sensors and Actuators A, vol. 99, no. 3, pp. 277-283, 2002.

[20] H. C. Seat and J. H. Sharp, " $\mathrm{Er}^{3+}+\mathrm{Yb}^{3+}$-codoped $\mathrm{Al}_{2} \mathrm{O}_{3}$ crystal fibres for high-temperature sensing," Measurement Science and Technology, vol. 14, no. 3, pp. 279-285, 2003.

[21] K. T. V. Grattan, Z. Y. Zhang, T. Sun, Y. H. Shen, L. M. Tong, and Z. C. Ding, "Sapphire-ruby single-crystal fibre for application in high temperature optical fibre thermometers: studies at temperatures up to $1500^{\circ} \mathrm{C}, "$ Measurement Science and Technology, vol. 12, no. 7, pp. 981-986, 2001.

[22] T. Takahashi, M. Katsuki, and Y. Mizutani, "Measurement of flame temperature by optical fiber thermometer," Experiments in Fluids, vol. 6, no. 8, pp. 514-520, 1988.

[23] Q. Zheng and K. Torii, "Response of optical fiber thermometer with blackbody cavity sensor," JSME International Journal, Series B, vol. 37, no. 3, pp. 588-595, 1994.

[24] M. R. Jones and D. G. Barker, "Use of blackbody optical fiber thermometers in high-temperature environments," Journal of Thermophysics and Heat Transfer, vol. 16, no. 3, pp. 306-312, 2002.

[25] P. R. N. Childs, J. R. Greenwood, and C. A. Long, "Review of temperature measurement," Review of Scientific Instruments, vol. 71, no. 8, pp. 2959-2978, 2000.

[26] S. W. Allison and G. T. Gillies, "Remote thermometry with thermographic phosphors: instrumentation and applications," Review of Scientific Instruments, vol. 68, no. 7, pp. 26152650, 1997.

[27] L. J. Dowell, G. T. Gillies, and S. W. Allison, "Measurements of lateral offset power losses in optical fibers scanning point sources," Optical Engineering, vol. 26, no. 6, pp. 547-552, 1987.

[28] S. W. Allison, G. T. Gillies, D. W. Magnuson, and T. S. Pagano, "Pulsed laser damage to optical fibers," Applied Optics, vol. 24, no. 19, pp. 3140-3145, 1985.

[29] S. W. Allison, M. R. Cates, G. T. Gillies, and B. W. Noel, "Fiber optic pulsed laser delivery system for remote measurements," Optical Engineering, vol. 26, no. 6, pp. 538-546, 1987.

[30] R. R. Dils and M. P. Moore, "Optical fiber thermometer measurements in automotive engine," Advances in Instrumentation, vol. 41, no. 3, pp. 1159-1175, 1988.

[31] G. W. Tregay, P. R. Calabrese, P. L. Kaplin, and M. J. Finney, "Optical fiber sensor for temperature measurement from 600 to $19008 \mathrm{C}$ in gas turbine engines," in Specialty Fiber Optic Systems for Mobile Platforms, vol. 1589 of Proceedings of SPIE, pp. 38-47, 1991.
[32] G. W. Tregay, P. R. Calabrese, M. J. Finney, and K. B. Stukey, "Durable fiber-optic sensors for gas temperature measurement in the hot section of turbine engines," in Fly-byLight, vol. 2295 of Proceedings of SPIE, pp. 156-162, San Diego, Calif, USA, July 1994.

[33] K. A. Wickersheim and R. V. Fuoropic, Thermometry: A Aew RF-Immune Technology, Alan Liss, New York, NY, USA, 1980.

[34] C. Ovren, M. Adoleson, and B. Hok, "Fiber optic systems for temperature and vibration measurement in industrial application," in Proceedings of the International Conference on Optical Techniques in Process Control, BHRA-British Hydromechanical Research Association, The Hague, The Netherlands, June 1983.

[35] K. A. James, W. H. Quick, and V. H. Strahan, "Fiber optics: the way to true digital sensors?" Control Engineering, vol. 26, no. 2, pp. 30-33, 1979.

[36] K. A. Wickersheim and M. H. Sun, "Fiberoptic thermometry and its application," Journal of Microwave Power and Electromagnetic Energy, vol. 22, no. 2, pp. 85-94, 1987.

[37] M. Sun, "Fiberoptic thermometry based on photoluminescent decay times," in Temperature: Its Measurement and Control in Science and Industry, vol. 6, pp. 715-719, American Institute of Physics, Melville, NY, USA, 1992.

[38] K. A. Wickersheim, "Application of fiber optic thermometry to the monitoring of widing temperatures in medium and large power transformers," in Fiber Optic and Laser Sensors IX, vol. 1584 of Proceedings of SPIE, pp. 3-14, Boston, Mass, USA, September 1991.

[39] L. J. Dowell and G. T. Gillies, "Errors caused by baseline offset and noise in the estimation of exponential lifetimes," Review of Scientific Instruments, vol. 62, no. 1, pp. 242-243, 1991.

[40] L. J. Dowell, G. T. Gillies, M. R. Cates, and S. W. Allison, "Precision limits of waveform recovery and analysis in a signal processing oscilloscope," Review of Scientific Instruments, vol. 58, no. 7, pp. 1245-1250, 1987.

[41] Z. Y. Zhang, K. T. V. Grattan, and A. W. Palmer, "Phaselocked detection of fluorescence lifetime," Review of Scientific Instruments, vol. 64, no. 9, pp. 2531-2540, 1993.

[42] Y. H. Shen, L. M. Tong, Y. Wang, and L. H. Ye, "Sapphire-fiber thermometer ranging from 20 to $1800^{\circ} \mathrm{C}$," Applied Optics, vol. 38, no. 7, pp. 1139-1143, 1999.

[43] B. E. Adams, "Optical fiber thermometry for use at high temperatures," in Temperature: Its Measurement and Control in Science and Industry, J. F. Schooley, Ed., vol. 6, pp. 739-743, American Institute of Physics, Melville, NY, USA, 1992. 

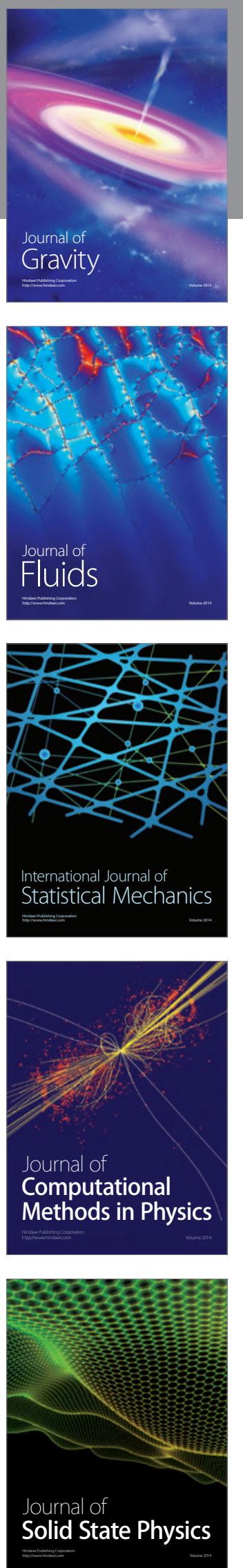

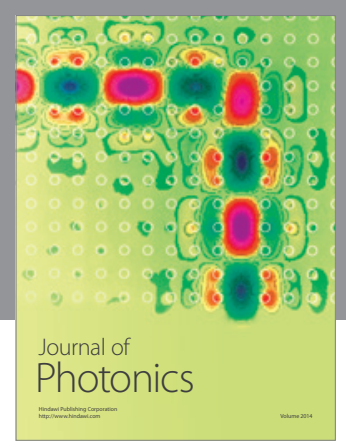

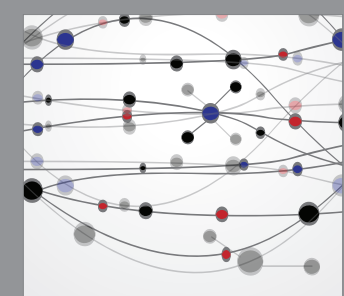

The Scientific World Journal
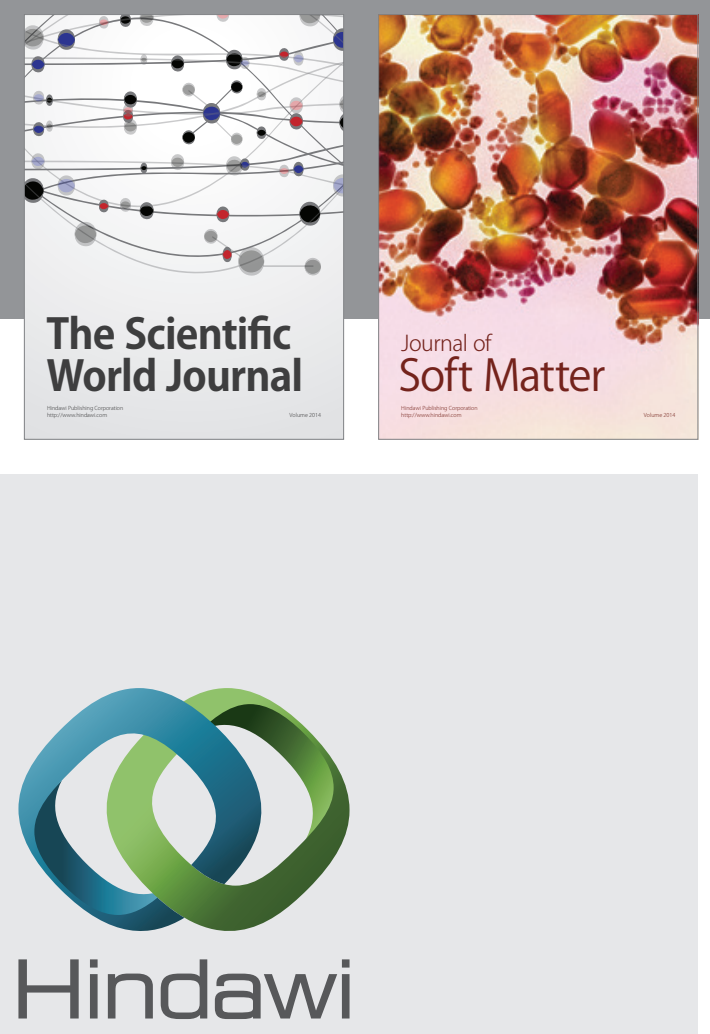

Submit your manuscripts at

http://www.hindawi.com
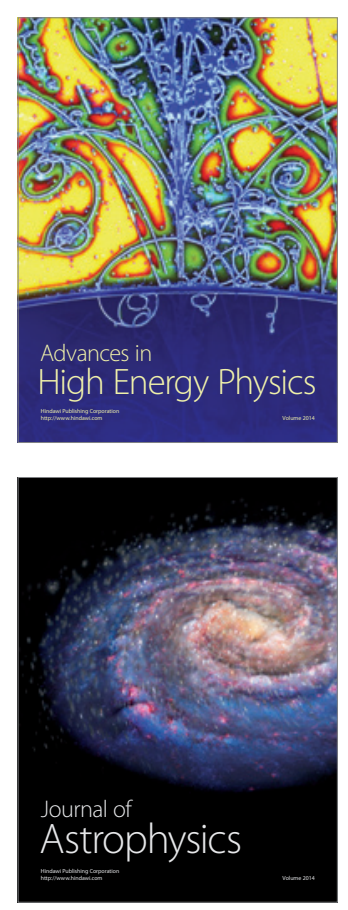
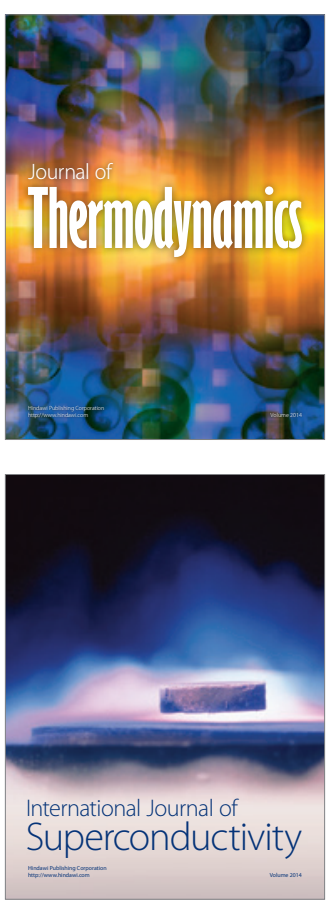
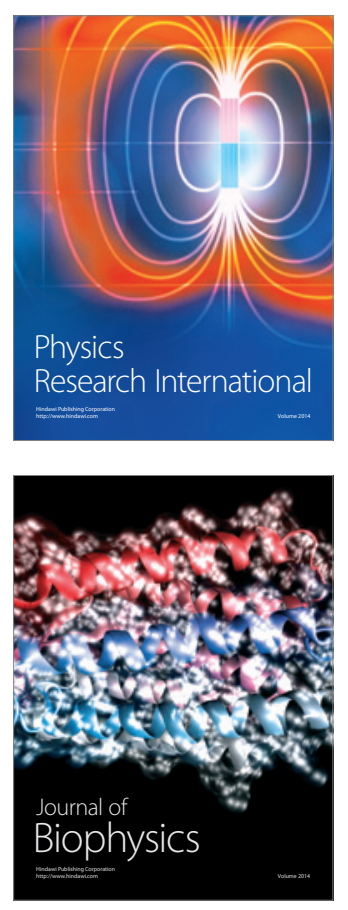
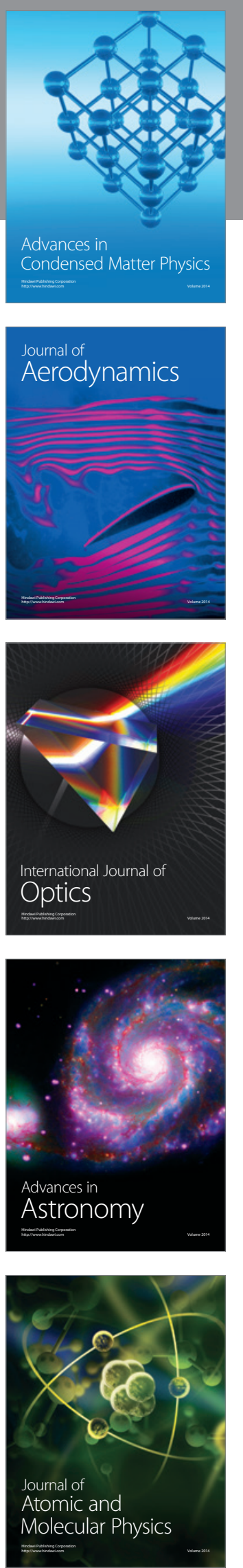